\title{
Comparing Euclidean and Hyperbolic Embeddings on the WordNet Nouns Hypernymy Graph
}

\author{
Sameer Bansal, Adrian Benton \\ Bloomberg \\ 731 Lexington Ave \\ New York, NY 10022 USA \\ $\{$ sbansal70, abenton10\}abloomberg. net
}

\begin{abstract}
Nickel and Kiela (2017) present a new method for embedding tree nodes in the Poincaré ball, and suggest that these hyperbolic embeddings are far more effective than Euclidean embeddings at embedding nodes in large, hierarchically structured graphs like the WordNet nouns hypernymy tree. This is especially true in low dimensions (Nickel and Kiela, 2017, Table 1). In this work, we seek to reproduce their experiments on embedding and reconstructing the WordNet nouns hypernymy graph. Counter to what they report, we find that Euclidean embeddings are able to represent this tree at least as well as Poincaré embeddings, when allowed at least 50 dimensions. We note that this does not diminish the significance of their work given the impressive performance of hyperbolic embeddings in very low-dimensional settings. However, given the wide influence of their work, our aim here is to present an updated and more accurate comparison between the Euclidean and hyperbolic embeddings.
\end{abstract}

\section{Introduction}

Nickel and Kiela (2017) introduced a method for learning embeddings in hyperbolic space for large, hierarchically structured objects like the WordNet nouns hypernymy graph. This work convincingly shows that across a range of embedding dimensions, from as low as 5 to as high as 200, hyperbolic embeddings consistently outperformed their Euclidean counterparts (Nickel and Kiela, 2017, Table 1). Illustrating the difference in performance at the highest experimental setting of 200 dimensions, the mean average precision (MAP) score for hyperbolic embeddings was shown to be around 5 times that of Euclidean for embedding nouns in the WordNet hypernymy graph. ${ }^{1}$ These experiments have been extremely influential, with the results on embedding the WordNet nouns hypernymy graph baselines often cited in later works on enhanced hyperbolic embeddings (De Sa et al., 2018; Ganea et al., 2018; Dhingra et al., 2018; López et al.,

\footnotetext{
${ }^{1}$ The embeddings were evaluated on a reconstruction task where a MAP score closer to 1 indicates better performance.
}

2019; Balazevic et al., 2019; Feyisetan et al., 2019; Chami et al., 2020). ${ }^{2}$

In this work, we reproduce the reconstruction error experiments on the WordNet noun hypernymy graph from Nickel and Kiela (2017). Counter to what they report, we find that Euclidean word embeddings are as effective at encoding the WordNet nouns graph as hyperbolic embeddings when given at least 50 dimensions. In fact, Euclidean embeddings with $\geq 100$ dimensions achieve lower reconstruction error over embeddings in the Lorentz model, an improved hyperbolic embedding method, which was published the following year (Nickel and Kiela, 2018).

The inability to reproduce the reported Euclidean experiments has been raised in several issues in the associated GitHub repository. ${ }^{3}$ This has also been acknowledged by the authors of the original study, who suggest that the original Euclidean embeddings were regularized in a way that may have hurt performance. ${ }^{4}$ However, the published manuscript has not been updated to reflect these problems with reproducing the Euclidean embedding baselines. As such, we hope that our reproduction will serve as a useful reference for those who are interested in exploring hyperbolic embeddings.

\section{Experimental Setup}

We use the source code released by the authors to carry out all experiments in this study, reusing the data processing, model training, and evaluation pipelines.

Dataset. Following Nickel and Kiela (2017), we embed the WordNet noun hierarchy (Fellbaum, 1998, WordNet-Nouns) in both Euclidean and hyperbolic space. Though the original study also published results on additional datasets, we restrict our focus to WordNet-Nouns, which exhibited a considerable gap in performance between embeddings.

Model training. Other than learning rate, we retain the default hyperparameters specified in the released source code, and train embeddings for 1,500 epochs.

\footnotetext{
${ }^{2}$ Nickel and Kiela (2017) has been cited over 500 times (source: www. semanticscholar.org).

${ }^{3}$ https: //github.com/facebookresearch/ poincare-embeddings/issues/35;68;72

${ }^{4}$ Author response on GitHub github.com/ facebookresearch/poincare-embeddings/ issues/35\#issuecomment-685174866
} 


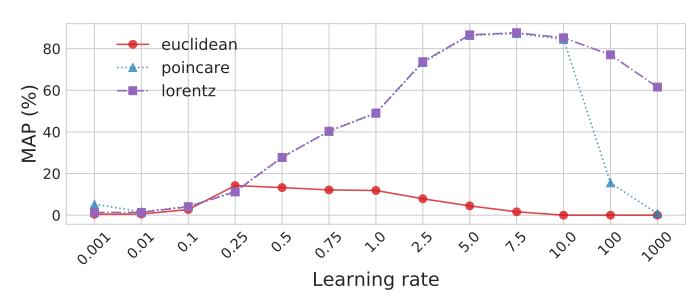

Figure 1: MAP of Euclidean, Poincaré, and Lorentz 20dimensional embeddings as a function of learning rate. MAP scores for Poincaré and Lorentz embeddings are very similar up to a learning rate of 10.0 .

The learning rate is tuned in the range $10^{[-2,3]}$ independently for each class of embeddings (Figure 1) with dimensionality fixed to 20, and selection based on loss after 200 epochs. We selected a learning rate of 0.5 for Euclidean and 5.0 for both Poincaré and Lorentz embeddings. In our initial experiments, we found that other hyperparameters, such as number of negative samples, also affected embeddings performance, but were less influential than learning rate. See Appendix A for details on the exact version of the codebase used in our experiments and how it was called. See the original study (Nickel and Kiela, 2017) for additional details on model training and evaluation.

Evaluation. Embeddings are evaluated under the original reconstruction error setting. For each hypernym pair $\langle u, v\rangle$ in the tree, rank all non-hypernyms along with $v$ by distance from $u$ in the embedded space. The fidelity to which a set of embeddings represents the tree is evaluated according to mean average precision (MAP) and mean rank (MR) of the positive example, $v$, averaged across rankings.

We focus on reconstruction error experiments as they are meant to highlight the capacity of each embedding space. The ability to generalize out of sample is an orthogonal question, however which we don't address in this work. This is mostly since the source code to reproduce these results from Nickel and Kiela (2017) has not yet been released, and the particular folds of heldout edges are also not provided. As such, we leave reproduction of the link prediction evaluation of Euclidean vs. hyperbolic embeddings to future work. ${ }^{5}$

\section{Results}

Table 1 shows the MAP and MR results for the WordNet-Nouns hierarchy reconstruction task. There are clear differences between the reproduced and reported performance for Euclidean embeddings. In the 50 dimensions setting, the reproduced Euclidean embeddings achieve a MAP score of 88.9 compared to 14 in the original study, and an MR score of 1.8 compared to 1,281 . In fact, this MR score for Euclidean embed-

\footnotetext{
${ }^{5}$ At the time of writing, there is an open GitHub issue to provide more details on the out of sample, link prediction evaluation https://github.com/ facebookresearch/poincare-embeddings / issues/10.
}

\begin{tabular}{ccccccr}
\hline dims & 5 & 10 & 20 & 50 & 100 & 200 \\
\hline \multicolumn{7}{c}{ Mean Average Precision \% (higher is better): } \\
$N \& K$ & 2.4 & 5.9 & 8.7 & 14 & 16.2 & 16.8 \\
ours & 2.7 & 4.5 & 11.2 & 88.9 & 91.7 & 92.2 \\
\hline \multicolumn{7}{c}{ Poincaré } \\
$N \& K$ & 82.8 & 86.5 & 85.5 & 86 & 85.7 & 87 \\
ours & 85.6 & 88.7 & 89.1 & 89.3 & 89.2 & 89.3 \\
\hline \multicolumn{7}{c}{ Lorentz } \\
$N \& K$ & 92.3 & 92.8 & - & - & - & - \\
ours & 87.4 & 88.6 & 89.5 & 89.3 & 89.4 & 89.4 \\
\hline \hline
\end{tabular}

\begin{tabular}{ccccrrr}
\hline \hline \multicolumn{7}{c}{ Mean Rank (lower is better): } \\
$N \& K$ & 3542 & 2286 & 1685 & 1281 & 1187 & 1157 \\
ours & 3646 & 1455 & 244 & 1.8 & 1.5 & 1.5 \\
\hline \multicolumn{7}{c}{ Poincaré } \\
$N \& K$ & 4.9 & 4.0 & 3.8 & 3.9 & 3.9 & 3.8 \\
ours & 6.8 & 5.6 & 5.2 & 4.9 & 4.9 & 4.9 \\
\hline \multicolumn{7}{c}{ Lorentz } \\
$N \& K$ & 3.1 & 2.9 & - & - & - & - \\
ours & 6.6 & 5.5 & 5 & 4.9 & 4.8 & 4.8 \\
\hline
\end{tabular}

Table 1: Mean average precision and mean rank for reconstructing the WordNet-Nouns hypernymy graph. $N \& K$ refers to best published results from Nickel and Kiela $(2017,2018)$.

\begin{tabular}{lrr}
\hline Embedding & MAP & MR \\
\hline Euclidean & $89.2 \pm 0.33$ & $1.8 \pm 0.00$ \\
Poincaré & $89.4 \pm 0.09$ & $4.9 \pm 0.01$ \\
Lorentz & $89.4 \pm 0.26$ & $4.9 \pm 0.10$ \\
\hline
\end{tabular}

Table 2: Mean and standard deviation of MAP and MR for 50-dimensional embeddings across three different random restarts.

dings at 50 dimensions is better than that achieved by Poincaré and Lorentz embeddings even with 200 dimensions. With greater than 50 dimensions, Euclidean embeddings outperform both Lorentz and Poincaré embeddings according to MR and MAP.

In contrast, the performance of hyperbolic embeddings remain stable across dimensionality and are similar between reproduced and reported results. The improved reconstruction error of Lorentz embeddings in prior work is likely due to a more comprehensive hyperparameter search.

We also found that performance is robust to random seed for all methods, with a standard deviation of less than a point for both MAP and MR score when training 50-dimensional Euclidean, Poincaré, or Lorentz embeddings (Table 2).

\section{Analysis}

In Section 3, we show it is possible to learn Euclidean embeddings that can reconstruct WordNet-Nouns more 


\begin{tabular}{lrrrrrr}
\hline dims & 5 & 10 & 20 & 50 & 100 & 200 \\
\hline \multirow{7}{*}{$\begin{array}{l}\text { Mean } \\
\text { Average }\end{array}$ Precision } & $\%$ & (higher is better): \\
ours: unit norm & 2.4 & 5.9 & 8.7 & 14.0 & 16.2 & 16.8 \\
\hline ours: no norm & 2.4 & 5.0 & 7.6 & 10.6 & 12.0 & 12.5 \\
\hline \multicolumn{7}{c}{ Mean Rank (lower is better): } \\
$N \& K$ & 2.7 & 4.5 & 11.2 & 88.9 & 91.7 & 92.2 \\
ours: unit norm & 3542 & 2286 & 1685 & 1281 & 1187 & 1157 \\
\hline ours: no norm & 3646 & 2275 & 1697 & 1276 & 1184 & 1159 \\
\hline
\end{tabular}

Table 3: MAP and MR for reconstructing the WordNet-Nouns hypernymy graph. Results from N\&K (Nickel and Kiela, 2017, 2018) and our reproduction with Euclidean embeddings constrained to unit norm are similar.

faithfully than similarly trained hyperbolic embeddings with at least 50 dimensions. In this section, we discuss possible reasons for the discrepancy between reported and reproduced Euclidean embeddings performance. We first posed this question to the authors themselves (Nickel and Kiela, 2017) who clarified on GitHub that the difference in performance for Euclidean embeddings in their published manuscript was due to a regularization method used at the time. They further added that in the released code they "disabled this regularization by default and it turned out to work better". 6 Follow-up questions regarding the details of this regularization method have yet to be addressed, at the time of writing. ${ }^{7}$

Constraining Euclidean Embedding Norm We speculate that the authors may have normalized the Euclidean embeddings to constrain them to lie within a unit 2-norm ball, similar to how Poincaré embeddings are trained (Nickel and Kiela, 2017, Section 3.1). Note that while projection into the unit ball is necessary to learn valid Poincaré embeddings, Euclidean embeddings require no such constraint.

The released source code actually supports projecting Euclidean embeddings into the unit ball after each iteration, but this is disabled by default, with the argument max_norm set to None. ${ }^{8}$ To test whether this constraint has an effect on embedding quality, we train Euclidean embeddings constrained to the unit ball by setting $m a x \_n o r m$ to 1 . Table 3 shows that reconstruction scores for Euclidean embeddings constrained to the unit ball are much closer to those published in Nickel

\footnotetext{
${ }^{6}$ Author response regarding difference in Euclidean performance https://github.com/ facebookresearch/poincare-embeddings / issues/35\#issuecomment-685261354

${ }^{7}$ Question seeking further information regarding regularization https://github.com/ facebookresearch/poincare-embeddings / issues/35\#issuecomment-685261354 and a relevant comment in issuecomment-735209781.

${ }^{8} \mathrm{~L} 2$ normalization disabled by default: https://github.com/facebookresearch/ poincare-embeddings/blob/4c7316b/hype/ manifolds/euclidean.py\#L16.
}

and Kiela $(2017,2018)$ than unconstrained Euclidean embeddings.

Varying Norm Constraints To explore the impact of norm constraints, we conduct further experiments on training 100 dimensional Euclidean embeddings. We vary the max_norm setting between 1 and 10, allowing the Euclidean embeddings to grow larger during training. As an alternative method for controlling the norm of the embeddings, we also vary the strength of an L2 penalty as an additional term in the loss. MAP and MR scores improve as the max_norm setting is increased, the norm constraint is relaxed (Table 4). In fact, setting max_norm to 5 yields Euclidean embeddings that achieve similar reconstruction performance as unconstrained embeddings.

We found that including an L2 regularization penalty in the loss has little effect on the final reconstruction scores. Thus, although we suspect unnecessary renormalization of the Euclidean embeddings may have caused the poor reconstruction performance reported in prior work, we defer to the authors of the original study for confirmation.

\begin{tabular}{lrr}
\hline Constraint & MAP & MR \\
\hline None (default) & 91.7 & 1.5 \\
L2 regularization $=0.01$ & 92.3 & 1.5 \\
L2 regularization $=1.0$ & 92.3 & 1.5 \\
L2 regularization $=100.0$ & 92.2 & 1.5 \\
max norm $=1$ & 11.2 & 1150.0 \\
max norm $=2$ & 38.3 & 40.3 \\
max norm $=5$ & 92.3 & 1.5 \\
max norm $=10$ & 92.3 & 1.5 \\
\hline$N \& K$ & 16.2 & 1187.3 \\
\hline
\end{tabular}

Table 4: MAP and MR for 100 dimensional embeddings. We include results with no regularization or constraints (row 1) and with L2 regularization of varying degree. $\max$ norm $=$ $k$ means that embeddings are projected back into a radius $k$ 2-norm ball after each iteration. $N \& K$ refers to best published results from Nickel and Kiela $(2017,2018)$ 


\section{Conclusion}

In our reproduction of the experiments in Table 1 of Nickel and Kiela (2017), we find that Euclidean actually outperform Poincaré embeddings when allowed a moderate number of dimensions. This is a realistic number of dimensions for typical non-contextual word embeddings, and a far lower dimensionality than subword token embeddings used in pretrained transformer language models. For example, released GloVe embeddings range from 50 to 300 dimensions (Pennington et al., 2014) and BERT base uses 768-dimensional subword embeddings (Devlin et al., 2019).

Nevertheless, the strong performance of hyperbolic embeddings in very low dimensions (less than 20) highlights their main strength: succinctly embedding nodes in hierarchically structured graphs with tight limitations on embedding size.

However, part of Nickel and Kiela (2017)'s impact came from the astounding gains from hyperbolic embeddings over Euclidean across a wide range of embedding widths. Subsequent application of Poincaré embeddings often report mixed results when using nonEuclidean vs. Euclidean embeddings in downstream tasks (Dhingra et al., 2018; López et al., 2019). We hope that this reproduction will serve as a valuable reference for others who are just beginning to explore hyperbolic embeddings.

\section{Acknowledgments}

We thank Maximilian Nickel and Douwe Kiela for their helpful feedback while carrying out this study and for making the source code available. We also thank the workshop organizers for providing an avenue for such work to be published and the anonymous reviewers whose feedback helped us improve this work. Thanks also to the GitHub users who reported similar issues in the source code repository, which motivated us to publish this study.

\section{References}

Ivana Balazevic, Carl Allen, and Timothy Hospedales. 2019. Multi-relational poincaré graph embeddings. In Proc. NeurIPS.

Ines Chami, Adva Wolf, Da-Cheng Juan, Frederic Sala, Sujith Ravi, and Christopher Ré. 2020. Lowdimensional hyperbolic knowledge graph embeddings. In Proc. ACL.

Christopher De Sa, Albert Gu, Christopher Ré, and Frederic Sala. 2018. Representation tradeoffs for hyperbolic embeddings. Proc. MLR, 80:4460.

Jacob Devlin, Ming-Wei Chang, Kenton Lee, and Kristina Toutanova. 2019. Bert: Pre-training of deep bidirectional transformers for language understanding. In Proc. NAACL, pages 4171-4186.

Bhuwan Dhingra, Christopher Shallue, Mohammad Norouzi, Andrew Dai, and George Dahl. 2018.
Embedding text in hyperbolic spaces. In Proc. (TextGraphs-12).

Christiane Fellbaum. 1998. WordNet: An Electronic Lexical Database.

Oluwaseyi Feyisetan, Tom Diethe, and Thomas Drake. 2019. Leveraging hierarchical representations for preserving privacy and utility in text. In Proc. ICDM.

Octavian Ganea, Gary Bécigneul, and Thomas Hofmann. 2018. Hyperbolic neural networks. In Proc. NeurIPS.

Federico López, Benjamin Heinzerling, and Michael Strube. 2019. Fine-grained entity typing in hyperbolic space. In Proc. (RepLANLP-2019).

Maximillian Nickel and Douwe Kiela. 2017. Poincaré embeddings for learning hierarchical representations. In Proc. NeurIPS.

Maximillian Nickel and Douwe Kiela. 2018. Learning continuous hierarchies in the lorentz model of hyperbolic geometry. In Proc. ICML.

Jeffrey Pennington, Richard Socher, and Christopher D Manning. 2014. Glove: Global vectors for word representation. In Proc. EMNLP, pages 1532-1543. 


\section{A Call to Poincaré Embedding Trainer}

Sample call to train embeddings and run reconstruction evaluation:

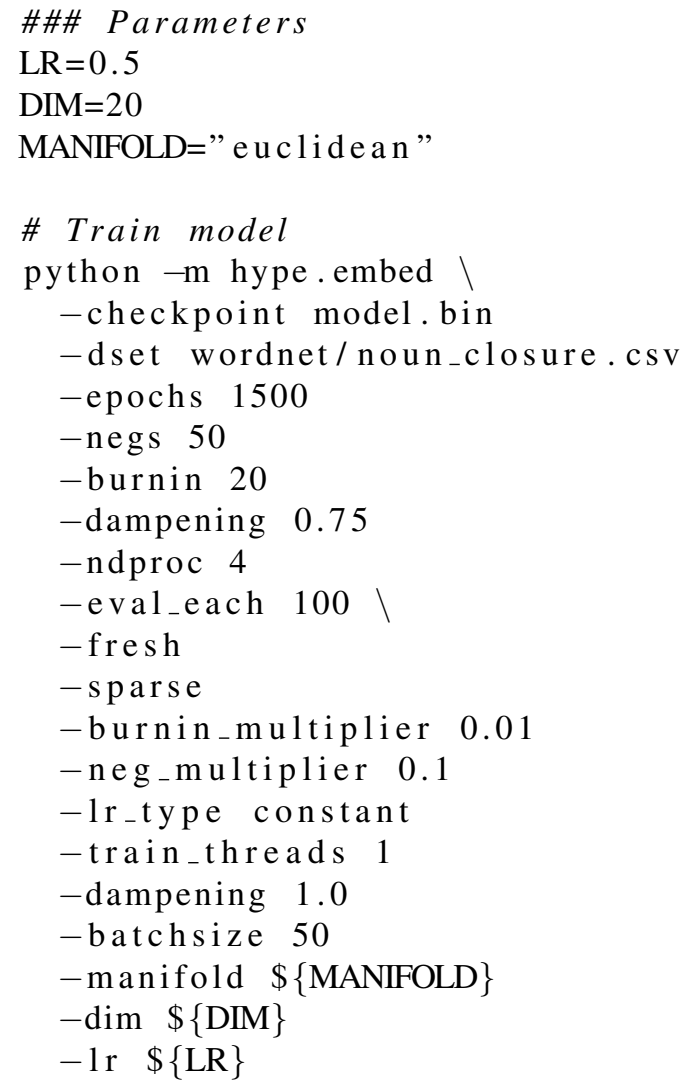

python reconstruction.py model.bin.1499

We use the poincare-embeddings implementation https://github. com/facebookresearch/ poincare-embeddings at commit 4 c 7316b14dce3b8 9e6a2d0c7994d418dffb42c94. 